TRANSACTIONS OF THE

AMERICAN MATHEMATICAL SOCIETY

Volume 353, Number 7 , Pages 2689-2703

S 0002-9947(01)02730-1

Article electronically published on March 12, 2001

\title{
ON THE SHELLABILITY OF THE ORDER COMPLEX OF THE SUBGROUP LATTICE OF A FINITE GROUP
}

\author{
JOHN SHARESHIAN
}

\begin{abstract}
We show that the order complex of the subgroup lattice of a finite group $G$ is nonpure shellable if and only if $G$ is solvable. A by-product of the proof that nonsolvable groups do not have shellable subgroup lattices is the determination of the homotopy types of the order complexes of the subgroup lattices of many minimal simple groups.
\end{abstract}

\section{INTRODUCTION}

We will show that the order complex of the subgroup lattice of a finite group $G$ is (nonpure) shellable if and only if $G$ is solvable. The proof of nonshellability in the nonsolvable case involves the determination of the homotopy type of the order complexes of the subgroup lattices of many minimal simple groups.

We begin with some history and basic definitions. It is assumed that the reader is familiar with some of the rudiments of algebraic topology and finite group theory. No distinction will be made between an abstract simplicial complex $\Delta$ and an arbitrary geometric realization of $\Delta$. Maximal faces of a simplicial complex $\Delta$ will be called facets of $\Delta$.

Definition 1.1. A simplicial complex $\Delta$ is shellable if the facets of $\Delta$ can be ordered $\sigma_{1}, \ldots, \sigma_{n}$ so that for all $1 \leq i<k \leq n$ there exists some $1 \leq j<k$ and $x \in \sigma_{k}$ such that $\sigma_{i} \cap \sigma_{k} \subseteq \sigma_{j} \cap \sigma_{k}=\sigma_{k} \backslash\{x\}$. The list $\sigma_{1}, \ldots, \sigma_{n}$ is called a shelling of $\Delta$.

Note that this definition does not require that $\Delta$ be pure. Pure shellable complexes are discussed in [1], and nonpure shellable complexes are introduced in [2].

Definition 1.2. Let $P$ be a partially ordered set. The order complex $\Delta(P)$ is the simplicial complex whose $k$-simplices are chains of length $k$ from $P$.

Note that a chain of length $k$ from a poset $P$ contains $k+1$ elements. We will call $P$ shellable if and only if $\Delta(P)$ is shellable.

Definition 1.3. Let $G$ be a finite group.

(1) $\mathcal{L}(G)$ is the lattice of subgroups of $G$.

(2) $\overline{\mathcal{L}(G)}:=\mathcal{L}(G) \backslash\{1, G\}$.

(3) $l(G)$ is the length of the longest chain in $\overline{\mathcal{L}(G)}$.

Received by the editors February 18, 1999 and, in revised form, May 1, 1999.

1991 Mathematics Subject Classification. Primary 06A11; Secondary 20E15.

(C)2001 American Mathematical Society 
It follows immediately from Definition 1.1 that $\mathcal{L}(G)$ is shellable if and only if $\overline{\mathcal{L}(G)}$ is shellable. We will call $G$ shellable if and only if $\overline{\mathcal{L}(G)}$ is shellable. As stated above, we will prove the following theorem.

Theorem 1.4. A finite group $G$ is shellable if and only if $G$ is solvable.

The relation between subgroup lattices and pure shellability is already wellunderstood. Note that for any poset $P$, the order complex $\Delta(P)$ is pure if and only if $P$ is graded, that is, every maximal chain in $P$ has the same length. In [10], Iwasawa showed that for a finite group $G, \mathcal{L}(G)$ is graded if and only if $G$ is supersolvable. In [14, Stanley introduced supersolvable lattices, which are lattices which share some important combinatorial properties with subgroup lattices of finite supersolvable groups. In [1], Björner showed that supersolvable lattices are shellable, thereby showing that the three conditions $G$ is supersolvable, $\Delta(\overline{\mathcal{L}(G)})$ is pure, and $\Delta(\overline{\mathcal{L}(G)})$ is shellable and pure are equivalent.

It follows from Theorem 1.4 and the work of Björner and Wachs in 2 that if $G$ is solvable then $\Delta(\overline{\mathcal{L}(G)})$ has the homotopy type of a wedge of spheres. The following stronger result was obtained by Kratzer and Thévenaz in 11.

Theorem 1.5 ([11, Corollaire 4.10]). Let $G$ be a finite solvable group with chief series $1=G_{0} \triangleleft G_{1} \triangleleft \ldots \triangleleft G_{r-1} \triangleleft G_{r}=G$. For $1 \leq i<r$, let $m_{i}$ be the number of complements to $G_{i} / G_{i-1}$ in $G / G_{i-1}$. Then $\Delta(\overline{\mathcal{L}(G)})$ has the homotopy type of a wedge of $m=\prod_{i=1}^{r-1} m_{i}$ spheres of dimension $r-2$.

It follows that the only reduced homology group of $\Delta=\Delta(\overline{\mathcal{L}(G)})$ which can be nontrivial is $\widetilde{H}_{r-2}(\Delta)$, which is free of dimension $m$. In [16], Thévenaz investigates the linear representation of $G$ on this homology group which is determined by the action of $G$ on $\Delta$ by conjugation. In doing so, he obtains an even stronger result than Theorem 1.5. Namely, assume that $m>0$ (where $m$ is as in Theorem 1.5), and fix a chief series $1=G_{0} \triangleleft \ldots \triangleleft G_{r}=G$. Let $\Gamma(G)$ be the set of all chains $1=C_{r}<C_{r-1}<\ldots<C_{0}=G$ such that each $C_{i}$ is a complement to $G_{i}$ in $G$. For $\gamma \in \Gamma(G)$, let $S_{\gamma}$ be the poset obtained by removing 1 and $G$ from the sublattice of $\mathcal{L}(G)$ generated by all the $G_{i}$ and all the $C_{i}$. Let $K(G)=\bigcup_{\gamma \in \Gamma(G)} S_{\gamma}$. Thévenaz shows (see Theorem 1.4 of [16]) that

- the identity embedding of $K(G)$ into $\overline{\mathcal{L}(G)}$ induces a homotopy equivalence of order complexes,

- $\Delta(K(G)) \simeq \bigvee_{\gamma \in \Gamma(G)} \Delta\left(S_{\gamma}\right)$, and

- for all $\gamma \in \Gamma(G), \Delta\left(S_{\gamma}\right)$ has the homotopy type of an $(r-2)$-sphere.

So, the spheres in the given wedge are indexed by the chains $\gamma$ of complements to the elements of a given chief series. Our proof that $\Delta(\overline{\mathcal{L}(G)})$ is shellable when $G$ is solvable uses the theory of recursive coatom orderings (see Section 5 of [2]), and we are unable to obtain the decomposition of Thévenaz or even the numerical result of Kratzer and Thévenaz as a corollary. However, Thévenaz' result suggests that the theory of EL-shellability (again, see section 5 of [2]) might be applied to the subgroup lattice of a solvable group, as in the following conjecture. As noted in [16], all chains $\gamma \in \Gamma(G)$ are maximal chains in $\mathcal{L}(G)$.

Conjecture 1.6. Let $G$ be a finite solvable group. Then $\mathcal{L}(G)$ admits an ELlabeling in which the falling maximal chains are exactly the elements of $\Gamma(G)$. 
I thank Anders Björner, Richard Lyons and Volkmar Welker for their encouragement and their helpful comments.

\section{Homotopy EQUiVALENCE OF ORDER COMPLEXES AND PROPERTIES OF SHELLABLE COMPLEXES}

It this section we will review some known results about order complexes and shellable complexes which will be used throughout the paper. The reader who is familiar with the main results in these areas can skip this section and refer back to it when necessary. We begin with some homotopy results of Quillen.

Definition 2.1. Let $P, Q$ be posets.

(1) A morphism $f: P \rightarrow Q$ is a function such that $x \leq_{P} y$ implies $f(x) \leq_{Q} f(y)$.

(2) If $f: P \rightarrow Q$ is a morphism, then for $y \in Q$ define

$$
f_{y}:=\{x \in P: f(x) \leq y\}
$$

and

$$
f^{y}:=\{x \in P: f(x) \geq y\} .
$$

Proposition $2.2([12,1.5])$. If a poset $P$ contains a unique minimal element or a unique maximal element, then $\Delta(P)$ is contractible.

Proposition 2.3 ([12, Proposition 1.6]). Let $f: P \rightarrow Q$ be a morphism of posets. If $\Delta\left(f_{y}\right)$ is contractible for all $y \in Q$ or $\Delta\left(f^{y}\right)$ is contractible for all $y \in Q$, then $f$ induces a homotopy equivalence between $\Delta(P)$ and $\Delta(Q)$.

Corollary 2.4. Let $P$ be a finite poset. Set

$$
S=\{x \in P: x \text { is covered by a unique element in } P\}
$$

and

$$
T=\{x \in P: x \text { covers a unique element in } P\} .
$$

Then for any $S^{\prime} \subseteq S$ and $T^{\prime} \subseteq T, \Delta(P)$ is homotopy equivalent to both $\Delta\left(P \backslash S^{\prime}\right)$ and $\Delta\left(P \backslash T^{\prime}\right)$.

Proof. Let $i: P \backslash S^{\prime} \hookrightarrow P$ be the identity embedding. Fix $y \in P$. If $y \notin S^{\prime}$ then $i^{y}=\left\{x \in P \backslash S^{\prime}: x \geq y\right\}$. If $y \in S^{\prime}$, let $z_{0}$ be the unique element of $P$ covering $y$. For $i \geq 0$, if $z_{i} \in S^{\prime}$ let $z_{i+1}$ be the unique element of $P$ covering $z_{i}$. Since $P$ is finite, there is a smallest $k$ such that $z_{k} \notin S^{\prime}$, and $i^{y}=\left\{x \in P \backslash S^{\prime}: x \geq z_{k}\right\}$. In any case, $\Delta\left(i^{y}\right)$ is contractible by Proposition 2.2, and $i$ induces a homotopy equivalence of order complexes by Proposition 2.3. The proof for $P \backslash T^{\prime}$ is similar.

Corollary 2.5. Let $L$ be a finite lattice and let $M$ be the sublattice of $L$ consisting of the minimum element $\hat{0}$, the maximum element $\hat{1}$, and all $x \in L$ such that $x=\bigwedge_{c \in C} c$ for some set $C$ of coatoms of $L$. For $X \in\{L, M\}$ let $\bar{X}=X \backslash\{\hat{0}, \hat{1}\}$. Then $\Delta(\bar{L})$ and $\Delta(\bar{M})$ are homotopy equivalent.

Proof. Let $i: \bar{M} \rightarrow \bar{L}$ be the identity embedding, and let $\mathcal{C}$ be the set of coatoms of $L$. For each $x \in \bar{L}$, let $\mathcal{C}(x)=\{c \in \mathcal{C}: x \leq c\}$. Then $\bigwedge_{c \in \mathcal{C}(x)} c$ is the unique minimum element of $i^{x}$. The corollary now follows from Propositions 2.2 and 2.3

Next we state a well-known collapsibility result (see [8]). 
Lemma 2.6. Let $\Delta$ be a simplicial complex and let $\tau$ be a face of $\Delta$ such that $\tau$ is properly contained in a unique facet of $\Delta$. Let $\Delta_{\tau}$ be the complex obtained by removing from $\Delta$ all faces containing $\tau$. Then $\Delta$ and $\Delta_{\tau}$ are homotopy equivalent.

The following results on shellable complexes appear in [2].

Definition 2.7. Let $\Delta$ be a finite simplicial complex.

(1) For a simplex $\sigma \in \Delta, d(\sigma):=\max \{|\tau|: \sigma \subseteq \tau \in \Delta\}$.

(2) For $0 \leq j \leq i, f_{i, j}$ is the number of simplices $\sigma \in \Delta$ such that $|\sigma|=j$ and $d(\sigma)=i$.

(3) For $0 \leq j \leq i, h_{i, j}:=\sum_{k=0}^{j}(-1)^{j-k}\left(\begin{array}{c}i-k \\ j-k\end{array}\right) f_{i, k}$.

Definition 2.8. Let $\Delta$ be a finite simplicial complex and let $\sigma_{1}, \ldots, \sigma_{n}$ be a shelling of $\Delta$.

(1) $R\left(\sigma_{k}\right):=\left\{x \in \sigma_{k}: \sigma_{k} \backslash\{x\} \subseteq \sigma_{i}\right.$ for some $\left.i<k\right\}$.

(2) $\Gamma_{j}:=\left\{\sigma_{k}:\left|\sigma_{k}\right|=j\right.$ and $\left.R\left(\sigma_{k}\right)=\sigma_{k}\right\}$.

Theorem 2.9 ([2, Theorems 3.4 and 4.1]). Let $\Delta$ be a finite simplicial complex and let $\sigma_{1}, \ldots, \sigma_{n}$ be a shelling of $\Delta$.

(1) Then $h_{i, j}$ is the number of $\sigma_{k}$ such that $\left|\sigma_{k}\right|=i$ and $\left|R\left(\sigma_{k}\right)\right|=j$. In particular, $h_{j, j}=\left|\Gamma_{j}\right|$ and $h_{i, j} \geq 0$ for all $i, j$.

(2) Also, $\Delta$ has the homotopy type of a wedge of spheres, consisting of $h_{j, j}$ spheres of dimension $j-1$ for each $j$. In particular, $\operatorname{dim}\left(\tilde{H}_{j-1}(\Delta)\right)=h_{j, j}$ is at most the number of facets of $\Delta$ having dimension $j-1$.

Definition 2.10. Let $\Delta$ be an abstract simplicial complex of finite dimension $d$. For each $0 \leq r \leq s \leq d$, define the complex

$$
\Delta^{(r, s)}:=\{\tau \in \Delta: \operatorname{dim}(\tau) \leq s \text { and } \tau \subseteq \sigma \text { for some } \sigma \in \Delta \text { with } \operatorname{dim}(\sigma) \geq r\} .
$$

Theorem 2.11 ([2, Theorem 2.9]). If $\Delta$ is a shellable complex of finite dimension $d$, then $\Delta^{(r, s)}$ is shellable for all $0 \leq r \leq s \leq d$.

Definition 2.12. Let $P$ be a finite poset with a unique minimum element $\hat{0}$ and a unique maximum element $\hat{1}$. We say that $P$ admits a recursive coatom ordering if $P=\{\hat{0}, \hat{1}\}$ or if there is an ordering $m_{1}, \ldots, m_{r}$ of the coatoms of $P$ which satisfies the following two properties.

(R) For all $j \in[r]$, the poset $P_{j}=\left[\hat{0}, m_{j}\right]$ admits a recursive coatom ordering in which the coatoms of $P_{j}$ which are contained in a poset $P_{i}=\left[\hat{0}, m_{i}\right]$ for some $i<j$ come before all other coatoms.

(S) For all $1 \leq i<k \leq r$ and $x \in P$, if $x \leq m_{i}$ and $x \leq m_{k}$, then there exist some $j<k$ and some coatom $w$ of $P_{k}$ such that $x \leq w \leq m_{j}$.

Since the posets we will work with are lattices, we can replace condition (S) in Definition 2.12 by the equivalent condition described below. The equivalence of the given conditions follows immediately from the definition of a lattice.

Proposition 2.13. Let $L$ be a finite lattice, and let $m_{1}, \ldots, m_{r}$ be an ordering of the coatoms of L. This ordering satisfies condition (S) of Definition [2.12 if and only if it satisfies the following condition.

(T) For all $1 \leq i<j \leq r$ there exists some $k<j$ such that $m_{i} \wedge m_{j} \leq m_{k} \wedge m_{j}$ and $m_{k} \wedge m_{j}$ is a coatom of $\left[\hat{0}, m_{j}\right]$. 
The next result, which follows immediately from Theorems 5.8 and 5.11 of [2], shows that in order to prove that $G$ is shellable it suffices to show that $\mathcal{L}(G)$ admits a recursive coatom ordering.

Theorem 2.14 (Björner-Wachs). Let $P$ be a finite poset with a unique minimum element $\hat{0}$ and a unique maximum element $\hat{1}$. If $P$ admits a recursive coatom ordering, then $P$ is shellable.

\section{Shellable groups are solvable}

In this section it will be shown that if a finite group $G$ is shellable then $G$ must be solvable. This result will be achieved by reducing the problem to the examination of the minimal simple groups. Recall that a minimal simple group is a nonabelian finite simple group all of whose proper subgroups are solvable. The reduction is achieved by applying an elementary result of Björner on shellable posets. Recall that a section of a finite group $G$ is a quotient group of a subgroup of $G$. If $G$ is a nonsolvable finite group, then some section of $G$ is a minimal simple group.

Proposition 3.1. If a finite group $G$ is shellable, then every section of $G$ is shellable.

Proof. Proposition 4.2 of [1] says that if a graded poset $P$ is shellable then every interval in $P$ is shellable. It is straightforward to adapt the proof of this proposition to the nongraded case. Now if $N \unlhd H \leq G$ then the interval $[N, H]$ in $\mathcal{L}(G)$ is isomorphic to $\mathcal{L}(H / N)$, and our proposition follows immediately.

The goal of this section is achieved by proving the following theorem.

Theorem 3.2. A minimal finite simple group is not shellable.

The minimal finite simple groups were determined by Thompson, well before the classification of finite simple groups was achieved. A list is given below.

Theorem 3.3 ([17, Corollary 1]). A finite simple group all of whose proper subgroups are solvable is isomorphic to one of the groups listed below.

(A) $L_{2}(p)$ with $p \geq 5$ prime and $p \not \equiv 1,4 \bmod 5$.

(B) $L_{2}\left(2^{p}\right)$ with $p$ prime.

(C) $L_{2}\left(3^{p}\right)$ with $p \geq 3$ prime.

(D) $S z\left(2^{p}\right)$ with $p \geq 3$ prime.

(E) $S L_{3}(3)$.

Note that $L_{2}(4) \cong L_{2}(5) \cong A_{5}$, where $A_{5}$ is the alternating group on five symbols. The structures of the subgroup lattices of the minimal simple groups are wellunderstood, and Theorem 3.2 will be proved by careful examination of each of the five classes of groups given above. We begin by determining all nontrivial subgroups of $G$ which are intersections of maximal subgroups when $G$ is one of the groups described in cases (A) through (D). In what follows, $Z_{n}$ denotes a cyclic group of order $n$ and $D_{n}$ denotes a dihedral group of order $n$. For a prime $p, E_{p^{a}}$ denotes an elementary abelian group of order $p^{a}$. For any groups $X$ and $Y, X . Y$ denotes a split extension of a group isomorphic to $X$ by a group of automorphisms of $X$ isomorphic to $Y$. Also, a.b will denote $X . Y$ if $X \cong Z_{a}$ and $Y \cong Z_{b} . S_{n}$ and $A_{n}$ denote the symmetric and alternating groups on $n$ letters, respectively. 
For a prime power $q$, all subgroups of $L_{2}(q)$ are listed in [4]. Those subgroups of $L_{2}(q)$ which are intersections of maximal subgroups are determined in [7]. The results when $L_{2}(q)$ is a minimal simple group are as follows.

Lemma 3.4. Let $p>7$ be a prime such that $L=L_{2}(p)$ is a minimal simple group. The maximal subgroups of $L$ are those on the following list.

- One conjugacy class of $\frac{|L|}{p-1} D_{p-1}$.

- One class of $\frac{|L|}{p+1} D_{p+1}$.

- One class of $p+1 p \cdot \frac{p-1}{2}$.

- Two classes of $\frac{|L|}{24} S_{4}$, if $p \equiv 1,7 \bmod 8$.

- One class of $\frac{|L|}{12} A_{4}$, if $p \equiv 3,5 \bmod 8$.

Furthermore, the nontrivial nonmaximal subgroups of $L$ which are intersections of maximal subgroups are those on the following list.

- One or two classes containing a total of $\frac{|L|}{8} D_{8}$, if $p \equiv 1,7 \bmod 8$.

- One or two classes containing a total of $\frac{|L|}{6} S_{3}$, if $p \equiv 1,7 \bmod 8$.

- Two classes of $\frac{|L|}{24} D_{4}$, if $p \equiv 1,7 \bmod 8$.

- One class of $\frac{|L|}{12} D_{4}$, if $p \equiv 3,5 \bmod 8$.

- One class of $\frac{|L|}{p-1} Z_{\frac{p-1}{2}}$.

- One class of $\frac{|L|}{v} Z_{3}$, where $v \in\{p+1, p-1\}$ and $3 \mid v$.

- One class of $\frac{|L|}{t} Z_{2}$, where $t \in\{p+1, p-1\}$ and $4 \mid t$.

The maximal subgroups of $A_{5}$ are contained in one conjugacy class of five $A_{4}$, one class of six $D_{10}$ and one class of ten $D_{6}$. The nontrivial nonmaximal subgroups of $A_{5}$ which are intersections of maximal subgroups are contained in one conjugacy class of ten $Z_{3}$ and one class of fifteen $Z_{2}$.

The maximal subgroups of $L_{2}(7)$ are contained in two conjugacy classes each containing seven $S_{4}$ and one class of eight 7.3. The nontrivial nonmaximal subgroups of $L_{2}(7)$ which are intersections of maximal subgroups are contained in one conjugacy class of twenty one $D_{8}$, one class of twenty eight $D_{6}$, two classes each containing seven $D_{4}$, one class containing twenty eight $Z_{3}$ and one class containing twenty one $Z_{2}$.

Lemma 3.5. Let $p$ be an odd prime and set $q=2^{p}$. The maximal subgroups of $L=L_{2}(q)$ are those on the following list.

- One conjugacy class of $\frac{|L|}{2(q-1)} D_{2(q-1)}$.

- One class of $\frac{|L|}{2(q+1)} D_{2(q+1)}$.

- One class of $q+1 E_{2^{p}} \cdot Z_{q-1}$.

Furthermore, the nontrivial nonmaximal subgroups of $L$ which are intersections of maximal subgroups are those on the following list.

- One class of $\frac{|L|}{2(q-1)} Z_{q-1}$.

- One class of $\frac{|L|}{q} Z_{2}$.

Lemma 3.6. Let $p$ be an odd prime and set $q=3^{p}$. The maximal subgroups of $L=L_{2}(q)$ are those on the following list.

- One conjugacy class of $\frac{|L|}{(q-1)} D_{q-1}$.

- One class of $\frac{|L|}{(q+1)} D_{q+1}$. 
- One class of $q+1 E_{3^{p}} \cdot Z_{\frac{q-1}{2}}$.

- One class of $\frac{|L|}{12} A_{4}$.

Furthermore, the nontrivial nonmaximal subgroups of $L$ which are intersections of maximal subgroups are those on the following list.

- One class of $\frac{|L|}{12} D_{4}$.

- One class of $\frac{|L|}{(q-1)} Z_{\frac{q-1}{2}}$.

- Two classes of $\frac{|L|}{q} Z_{3}$.

- One class of $\frac{|L|}{q+1} Z_{2}$.

The maximal subgroups of the Suzuki groups are determined in [15]. Using further information from [15], one can determine the nonmaximal subgroups of $S z\left(2^{p}\right)$ which are intersections of maximal subgroups. The results are stated below.

Lemma 3.7. Let $p$ be an odd prime. Set $q=2^{p}$ and $r=\sqrt{2 q}$. The maximal subgroups of $G=S z(q)$ are those on the following list.

- One conjugacy class of $\frac{|G|}{q^{2}(q-1)} S \cdot Z_{q-1}$, where $S \in S y l_{2}(G)$.

- One class of $\frac{|G|}{2(q-1)} D_{2(q-1)}$.

- One class of $\frac{|G|}{4(q+r+1)}(q+r+1) \cdot 4$.

- One class of $\frac{|G|}{4(q-r+1)}(q-r+1) \cdot 4$.

Furthermore, the nontrivial nonmaximal subgroups of $G$ which are intersections of maximal subgroups are those on the following list.

- One class of $\frac{|G|}{2(q-1)} Z_{q-1}$.

- One class of $\frac{|G|}{2 q} Z_{4}$.

- One class of $\frac{|G|}{q^{2}} Z_{2}$.

We will now use the four lemmas just stated to determine the homotopy type of $\Delta(\overline{\mathcal{L}(G)})$ in cases (A) through (D) of Theorem 3.3 except in the case $G=L_{2}(p)$ with $p \equiv 1,7 \bmod 8$.

Lemma 3.8. Let $G$ be a minimal simple group isomorphic to one of $L_{2}(p)$ with $p \equiv 3,5 \bmod 8, L_{2}\left(2^{p}\right), L_{2}\left(3^{p}\right)$ or $S z\left(2^{p}\right)$. Then $\Delta(\overline{\mathcal{L}(G)})$ has the homotopy type of a wedge of $|G| 1$-spheres.

Proof. In each of the given cases, it is known that $\tilde{\chi}(\Delta(\overline{\mathcal{L}(G)}))=-|G|$ (see $[9]$ for $L_{2}(p)$, [6] or [7] for $L_{2}\left(2^{p}\right)$, 7] or [13] for $L_{2}\left(3^{p}\right)$ and [13] for $\left.S z\left(2^{p}\right)\right)$. Thus it is sufficient to show that $\Delta(\overline{\mathcal{L}(G)})$ is connected and homotopy equivalent to a 1-dimensional complex. To show that $\Delta(\overline{\mathcal{L}(G)})$ is connected, it suffices to show that if $X$ and $Y$ are maximal subgroups of $G$ then there is a path in the 1-skeleton of $\Delta(\overline{\mathcal{L}(G)})$ between (the vertices corresponding to) $X$ and $Y$. First note that if $A$ and $B$ are subgroups of $G$ of order two then $\langle A, B\rangle$ is a proper dihedral subgroup of $G$. Thus there is a path from $A$ to $B$. It follows that all maximal subgroups of $G$ having even order are in the same connected component of the 1-skeleton. If $X \leq G$ is a maximal subgroup of odd order, then it follows from the four lemmas above that either $G=L_{2}(p)$ with $p \equiv 3 \bmod 4$ and $X=p \cdot \frac{p-1}{2}$, or $G=L_{2}\left(3^{p}\right)$ and $X=E_{3^{p}} \cdot Z_{\frac{3^{p}-1}{2}}$. In either case, there exists a dihedral maximal subgroup $D<G$ 
such that $D \cap X \neq 1$. Thus there is a path from $X$ to $D$, and it follows that the 1 -skeleton is connected.

Let $\mathcal{P}$ be the subposet of $\overline{\mathcal{L}(G)}$ consisting of those nontrivial proper subgroups of $G$ which are intersections of maximal subgroups, of course including the maximal subgroups themselves. By Corollary 2.5$] \Delta(\overline{\mathcal{L}(G)})$ and $\Delta(\mathcal{P})$ have the same homotopy type, so it suffices to show that $\Delta(\mathcal{P})$ is homotopy equivalent to a 1-dimensional complex. By Lemma 3.5] if $G=L_{2}\left(2^{p}\right)$ then $\Delta(\mathcal{P})$ is already 1-dimensional, so it remains to examine the other three cases.

First assume $G=S z\left(2^{p}\right)$. It is shown in [15] that $G$ has one conjugacy class of involutions, so by Lemma 3.7 every subgroup of $G$ having order two is in $\mathcal{P}$. It follows that every element of $\mathcal{P}$ isomorphic to $Z_{4}$ covers a unique element of $\mathcal{P}$. Let $\mathcal{P}_{0}$ be the poset obtained by removing all subgroups isomorphic to $Z_{4}$ from $\mathcal{P}$. By Corollary [2.4 $\Delta(\mathcal{P})$ and $\Delta\left(\mathcal{P}_{0}\right)$ have the same homotopy type. By Lemma 3.7 $\Delta\left(\mathcal{P}_{0}\right)$ is 1-dimensional.

Finally, assume $G=L_{2}(q)$, where either $q$ is prime and satisfies the conditions of the lemma or $q=3^{p}$ with $p$ an odd prime. By Lemmas 3.4 and 3.6, every facet in $\Delta(\mathcal{P})$ has dimension one or two. Furthermore, if $\sigma=X<Y<Z$ is a 2-simplex in $\Delta(\mathcal{P})$ then exactly one of the following cases holds.

1. $X \cong Z_{2}, Y \cong D_{4}$ and $Z \cong A_{4}$.

2. $X \cong Z_{3}, Y \cong Z_{\frac{q-1}{2}}, Z \cong D_{q-1}, q$ is prime and $q \equiv 1 \bmod 3$.

3. $X \cong Z_{3}, Y \cong Z_{\frac{q-1}{2}}, Z \cong q \cdot \frac{q-1}{2}, q$ is prime and $q \equiv 1 \bmod 3$.

4. $X \cong Z_{2}, Y \cong Z_{\frac{q-1}{2}}, Z \cong q \cdot \frac{q-1}{2}, q$ is prime and $q \equiv 1 \bmod 4$.

5. $X \cong Z_{2}, Y \cong D_{4}$ and $Z \cong D_{n}$, where $n \in\{q-1, q+1\}$ with $4 \mid n$, and $X$ is not the center of $Z$.

6. $X \cong Z_{2}, Y \cong Z_{\frac{q-1}{2}}, Z \cong D_{q-1}, q$ is prime and $q \equiv 1 \bmod 4$.

7. $X \cong Z_{2}, Y \cong D_{4}$ and $Z \cong D_{n}$, where $n \in\{q-1, q+1\}$ with $4 \mid n$, and $X$ is the center of $Z$.

In the first five cases above, $\sigma$ is the unique facet of $\Delta(\mathcal{P})$ containing $\tau(\sigma)=$ $X<Z$. Let $\Delta_{0}$ be the complex obtained by removing from $\Delta(\mathcal{P})$ all pairs $\sigma, \tau(\sigma)$ where $\sigma$ is one of the 2-simplices described in the first five cases above. By Lemma 2.6. $\Delta(\mathcal{P})$ and $\Delta_{0}$ are homotopy equivalent. Now let $\sigma$ be as in the sixth or seventh case above. Then $\sigma$ is the unique facet in $\Delta_{0}$ containing $\tau(\sigma)=Y<Z$. Let $\Delta_{1}$ be the complex obtained by removing from $\Delta_{0}$ all pairs $\sigma, \tau(\sigma)$ where $\sigma$ is one of the 2-simplices described in the sixth or seventh case above. Again by Lemma 2.6. $\Delta_{0}$ is homotopy equivalent to the 1-dimensional complex $\Delta_{1}$, and the lemma follows.

Corollary 3.9. Let $G$ be a minimal simple group isomorphic to one of $L_{2}(p)$ with $p \equiv 3,5 \bmod 8$ and $p>5, L_{2}\left(2^{p}\right)$ with $p$ an odd prime, $L_{2}\left(3^{p}\right)$ or $S z\left(2^{p}\right)$. Then $\Delta(\overline{\mathcal{L}(G)})$ is not shellable.

Proof. By Theorem 2.9 and Lemma 3.8 , if $\Delta(\overline{\mathcal{L}(G)})$ is shellable then it contains at least $|G|$ 1-dimensional facets. We will examine each possibility for $G$ using Lemmas 3.4 3.5, 3.6 and 3.7, and show that this condition cannot hold. Note that a 1-dimensional facet in $\Delta(\overline{\mathcal{L}(G)})$ is a chain $X<Y$ such that $Y$ is a maximal subgroup of $G, X$ is a maximal subgroup of $Y$ and $|X|$ is prime.

If $G=L_{2}(p)$ with $p \equiv 3,5 \bmod 8$, then $\Delta(\overline{\mathcal{L}(G)})$ contains $\frac{|G|}{3}$ 1-dimensional facets $X<Y$ with $X \cong Z_{3}$ and $Y \cong A_{4}$. There are no other 1-dimensional facets 
in $\Delta(\overline{\mathcal{L}(G)})$ unless $\frac{p-1}{2}$ or $\frac{p+1}{2}$ is prime. Since $p>5$, at most one of these two numbers can be prime. If $\frac{p-1}{2}$ is prime then the remaining 1-dimensional facets in $\Delta(\overline{\mathcal{L}(G)})$ are $\frac{|G|}{2}$ chains $X<Y$ with $X \cong Z_{2}$ and $Y \cong D_{p-1}, \frac{|G|}{p-1}$ chains $X<Y$ with $X \cong Z_{\frac{p-1}{2}}$ and $Y \cong D_{p-1}, \frac{2|G|}{p-1}$ chains $X<Y$ with $X \cong Z_{\frac{p-1}{2}}$ and $Y \cong p \cdot \frac{p-1}{2}$, and $\frac{2|G|}{p(p-1)}$ chains $X<Y$ with $X \cong Z_{p}$ and $Y \cong p \cdot \frac{p-1}{2}$. It follows that if there are at least $|G| 1$-facets then $p<20$. However, the only primes $p<20$ with $p \equiv 3,5 \bmod 8$ and $\frac{p-1}{2}$ prime are 5 and 11 , and $L_{2}(11)$ contains $A_{5}$ and is therefore not a minimal simple group. If $\frac{p+1}{2}$ is prime, then the remaining 1-dimensional facets in $\Delta(\overline{\mathcal{L}(G)})$ are $\frac{|G|}{2}$ chains $X<Y$ with $X \cong Z_{2}$ and $Y \cong D_{p+1}$, and $\frac{|G|}{p+1}$ chains with $X \cong Z_{\frac{p+1}{2}}$ and $Y \cong D_{p+1}$. Since $p>5$, there are less than $|G| 1$-dimensional facets.

If $G=L_{2}(q)$ where $q=2^{p}$ with $p$ an odd prime, then $\Delta(\overline{\mathcal{L}(G)})$ contains no 1-dimensional facets unless $q-1$ is prime. In that case, the 1-dimensional facets in $\Delta(\overline{\mathcal{L}(G)})$ are $\frac{|G|}{2}$ chains $X<Y$ with $X \cong Z_{2}$ and $Y \cong D_{2(q-1)}, \frac{|G|}{2(q-1)}$ chains $X<Y$ with $X \cong Z_{q-1}$ and $Y \cong D_{2(q-1)}$, and $\frac{|G|}{q-1}$ chains $X<Y$ with $X \cong Z_{q-1}$ and $Y \cong E_{2^{p}} \cdot Z_{q-1}$. The total number of 1-dimensional facets in this case is less than $|G|$.

If $G=L_{2}(q)$ where $q=3^{p}$ with $p$ an odd prime, then $\Delta(\overline{\mathcal{L}(G)})$ contains $\frac{|G|}{3}$ facets $X<Y$ with $X \cong Z_{3}$ and $Y \cong A_{4}$, and no other 1-dimensional facets unless $\frac{q-1}{2}$ is prime. If $\frac{q-1}{2}$ is prime, then the additional 1-dimensional facets of $\Delta(\overline{\mathcal{L}(G)})$ are $\frac{|G|}{2}$ chains $X<Y$ with $X \cong Z_{2}$ and $Y \cong D_{q-1}, \frac{|G|}{q-1}$ chains $X<Y$ with $X \cong Z_{\frac{q-1}{2}}$ and $Y \cong D_{q-1}$, and $\frac{2|G|}{q-1}$ chains $X<Y$ with $X \cong Z_{\frac{q-1}{2}}$ and $Y \cong E_{3^{p}} \cdot Z_{\frac{q-1}{2}}$. Since $q \geq 27$, there are less than $|G|$ 1-dimensional facets.

If $G=S z(q)$ where $q=2^{p}$ with $p$ an odd prime, then $\Delta(\overline{\mathcal{L}(G)})$ has no 1dimensional facets unless $q-1$ is prime. If $q-1$ is prime then the 1-dimensional facets of $\Delta(\overline{\mathcal{L}(G)})$ are $\frac{|G|}{2}$ chains $X<Y$ with $X \cong Z_{2}$ and $Y \cong D_{2(q-1)}$, and $\frac{|G|}{2(q-1)}$ chains $X<Y$ with $X \cong Z_{q-1}$ and $Y \cong D_{2(q-1)}$. Here we have used the fact, stated in [15], that if $S \in S l_{2}(G)$ then $S$ is not abelian. Thus $S$ has a characteristic nontrivial proper subgroup and $S . Z_{q-1}$ has no maximal subgroup of order $q-1$. In any case, $\Delta(\overline{\mathcal{L}(G)})$ has less than $|G|$ 1-dimensional facets, and we are done.

Lemma 3.10. If $G=A_{5}$ then $\Delta(\overline{\mathcal{L}(G)})$ is not shellable.

Proof. Write $\Delta$ for $\Delta(\overline{\mathcal{L}(G)})$. If $\Delta$ is shellable then by Theorem $\left[2.11\right.$ so is $\Delta^{(2,2)}$. By Lemma 3.4, the facets of $\Delta^{(2,2)}$ are 15 chains $X<Y<Z$ with $X \cong Z_{2}, Y \cong D_{4}$ and $Z \cong A_{4}$. If $\sigma$ and $\tau$ are two such facets then $|\sigma \cap \tau| \leq 1$, as any involution in $A_{5}$ fixes exactly one point in the natural representation and each $A_{4}$ is the stabilizer of a point. It follows immediately from Definition 1.1 that $\Delta^{(2,2)}$ is not shellable.

It remains to examine the minimal simple groups $L_{2}(p)$ with $p \equiv 1,7 \bmod 8$, along with $S L_{3}(3)$.

Lemma 3.11. Let $G=L_{2}(p)$ be a minimal simple group with $p \equiv 1,7 \bmod 8$. Then $\tilde{H}_{1}(\Delta(\overline{\mathcal{L}(G)})) \neq 0$.

Proof. Let $\mathcal{P}$ be the subposet of $\overline{\mathcal{L}(G)}$ consisting of those groups which are intersections of maximal subgroups of $G$. By Corollary 2.5, it is sufficient to show that $\tilde{H}_{1}(\Delta(\mathcal{P})) \neq 0$. Set $r=\frac{p-1}{2}$. By Lemma [3.4, $\Delta(\mathcal{P})$ contains 1-simplices $X<Y$ 
with $X \cong Z_{r}$ and $Y \cong p . r$. Furthermore, any facet of $\Delta(\mathcal{P})$ properly containing one of these 1-simplices is of the form $\sigma=W<X<Y$ with $W \cong Z_{a}$, where $a \in\{2,3\}$ and $a \mid r$. For such a facet $\sigma$, let $\tau(\sigma)=W<Y$. Then $\sigma$ is the unique facet of $\Delta(\mathcal{P})$ containing $\tau(\sigma)$. Let $\Delta_{0}$ be the complex obtained by removing all pairs $\sigma, \tau(\sigma)$ described above from $\Delta(\mathcal{P})$. By Lemma 2.6 $\Delta(\mathcal{P})$ and $\Delta_{0}$ have the same homotopy type, so it suffices to show that $\tilde{H}_{1}\left(\Delta_{0}\right) \neq 0$. Let $\Delta_{1}$ be the subcomplex of $\Delta_{0}$ whose facets are all chains $X<Y$ with $X \cong Z_{r}$ and $Y \cong p$.r. Then $\Delta_{1}$ is a graph with $p+1+\frac{p(p+1)}{2}$ vertices and $p(p+1)$ edges. Since $p>2, \Delta_{1}$ has more edges than vertices, and is not a forest. Therefore, $\tilde{H}_{1}\left(\Delta_{1}\right) \neq 0$. Since every 1-simplex in $\Delta_{1}$ is a facet in $\Delta_{0}, \tilde{H}_{1}\left(\Delta_{0}\right) \neq 0$ and we are done.

Corollary 3.12. Let $G=L_{2}(p)$ be a minimal simple group with $p \equiv 1,7 \bmod 8$. Then $\Delta(\overline{\mathcal{L}(G)})$ is not shellable.

Proof. By Lemma 3.11 and Theorem 2.9 $(2)$, if $\Delta(\overline{\mathcal{L}(G)})$ is shellable then it contains some 1-dimensional facets. By Lemma 3.4 $\Delta(\overline{\mathcal{L}(G)})$ contains 1-dimensional facets if and only if either $\frac{p-1}{2}$ or $\frac{p+1}{2}$ is prime. We will examine both of these possibilities. From now on, we write $\Delta$ for $\Delta(\overline{\mathcal{L}(G)})$. Let $d$ be the dimension of the largest facet in $\Delta$, so $d=l(G)-1$.

First assume that $\frac{p+1}{2}$ is prime, and set $r=\frac{p-1}{2}$. Note that in this case, $p \equiv$ $1 \bmod 8$, so $2 \mid r$. By Lemma 3.4 $\Delta^{(2, d)}$ is the order complex of the ideal in $\overline{\mathcal{L}(G)}$ generated by maximal subgroups of $G$ isomorphic to $D_{2 r}, p . r$ or $S_{4}$. Let $\mathcal{P}$ be the subposet of $\overline{\mathcal{L}(G)}$ consisting of those subgroups of $G$ which are intersections of these maximal subgroups. Then $\Delta^{(2, d)}$ and $\Delta(\mathcal{P})$ have the same homotopy type by Corollary 2.5 and $\Delta(\mathcal{P})$ contains the chains $X<Y$ with $X \cong Z_{r}$ and $Y \cong p . r$ described in Lemma 3.11. The same argument used in the proof of that lemma shows that $\tilde{H}_{1}\left(\Delta^{(2, d)}\right) \neq 0$. It now follows from Theorem 2.9(2) and the fact that $\Delta^{(2, d)}$ has no 1-dimensional facets that $\Delta^{(2, d)}$ is not shellable. By Theorem 2.11 $\Delta$ is not shellable.

Now, assume that $\frac{p-1}{2}$ is prime, and set $s=\frac{p+1}{2}$. Note that in this case, $p \equiv 7 \bmod 8$, so $8 \mid 2 s$. It follows that exactly one of the following three cases holds.

1. $p+1=2 s$ has at least five prime factors, counting multiplicities.

2. $3 \mid p-1$, so $p=7$.

3. $p+1=24$, so $p=23$.

If $2 s$ has at least five prime factors, then by Lemma $3.4 \Delta^{(d, d)}$ is the order complex of the ideal in $\overline{\mathcal{L}(G)}$ generated by the maximal subgroups of $G$ isomorphic to $D_{2 s}$. Let $\mathcal{P}$ be the subposet of $\overline{\mathcal{L}(G)}$ consisting of those groups which are intersections of these maximal subgroups, so $\Delta^{(d, d)}$ and $\Delta(\mathcal{P})$ have the same homotopy type by Corollary 2.5. By Lemma $3.4 \mathcal{P}$ consists of those subgroups of $G$ isomorphic to $Z_{2}, D_{4}$ or $D_{2 s}$. Let $\mathcal{Q}$ be the subposet of $\mathcal{P}$ consisting of those subgroups of $G$ isomorphic to $D_{4}$ or $Z_{2}$, and let $i: \mathcal{Q} \hookrightarrow \mathcal{P}$ be the identity embedding. Note that for each $X \in \mathcal{P}, i_{X}$ is the poset of nontrivial elementary abelian 2-subgroups of $X$. Also, note that $O_{2}(X) \neq 1$, that is, each $X \in \mathcal{P}$ contains a nontrivial normal 2-subgroup. By [12, Lemma 2.2], $i_{X}$ is contractible for all $X \in \mathcal{P}$. Thus by Proposition 2.3, $\Delta(\mathcal{P})$ and $\Delta(\mathcal{Q})$ have the same homotopy type. Now $\Delta(\mathcal{Q})$ is a graph with $\frac{|G|}{12}+\frac{|G|}{2 s}$ vertices and $\frac{|G|}{4}$ edges. Since there are more edges than vertices, $\Delta(\mathcal{Q})$ is not a forest and $\tilde{H}_{1}(\Delta(\mathcal{Q})) \neq 0$. Thus $\tilde{H}_{1}\left(\Delta^{(d, d)}\right) \neq 0$, and since 
$\Delta^{(d, d)}$ has no 1-dimensional facets, it is not shellable by Theorem 2.9(2). Now $\Delta$ is not shellable by Theorem 2.11.

If $p=7$ then by Lemma 3.4 $\Delta^{(3,3)}$ is the order complex of the subposet $\mathcal{P}$ of $\overline{\mathcal{L}(G)}$ consisting of those subgroups of $G$ isomorphic to $Z_{2}, Z_{4}, D_{4}, D_{8}, A_{4}$ or $S_{4}$. Note that $O_{2}(X) \neq 1$ for each $X \in \mathcal{P}$. Now as in the above paragraph, we see that $\Delta^{(3,3)}$ and $\Delta(\mathcal{Q})$ have the same homotopy type, where $\mathcal{Q}$ is the subposet of $\overline{\mathcal{L}(G)}$ consisting of those subgroups of $G$ isomorphic to $Z_{2}$ or $D_{4}$, and that $\tilde{H}_{1}(\Delta(\mathcal{P})) \neq 0$. As above, it follows that $\Delta$ is not shellable.

If $p=23$ the situation is more complicated, but it can be shown that $\Delta$ is not shellable either by using Lemma 2.6 to show that $\Delta^{(3,3)}$ has the same homotopy type as a noncontractible 2-dimensional complex, or by calculating that $h_{4,3}=-3,040$ and applying Theorem 2.9 (1). This last calculation can be done by hand.

Lemma 3.13. If $G=S L_{3}(3)$ then $\Delta(\overline{\mathcal{L}(G)})$ is not shellable.

Proof. Write $\Delta$ for $\Delta(\overline{\mathcal{L}(G)})$, and let $d=l(G)-1$ be the dimension of the largest facet of $\Delta$. We will see that $\Delta^{(d, d)}$ is not shellable and apply Theorem 2.11. Let $V$ be a 3 -dimensional vector space over $\mathbf{F}_{3}$ on which $G$ acts naturally. It is known that the only subgroups of $G$ which are of prime index in $G$ are the stabilizers in $G$ of the nontrivial proper subspaces of $V$ (see for example [3]). For any finite solvable group $H, l(H)+2$ is equal to the number of prime factors of $|H|$, counting multiplicities. It follows that the facets of $\Delta^{(d, d)}$ are chains $X_{0}<X_{1}<\ldots<X_{d}$ such that $X_{d}$ is a subspace stabilizer and $\left[X_{i}: X_{i-1}\right]$ is prime for $1 \leq i \leq d$.

It is known that $G$ acts 2-transitively on the set of 131 -spaces in $V$ and on the set of 132 -spaces in $V$. Let $M<G$ be the stabilizer of a fixed 1-space $W<V$. If $K$ is the stabilizer of another 1-space, then by 2-transitivity $[M: K \cap M]=12$. Also by 2-transitivity, $M$ acts transitively on the set of four 2-spaces in $V$ which contain $W$, so if $K$ is the stabilizer of such a 2-space then $[M: K \cap M]=4$. It can be shown using elementary linear algebra that $M$ also acts transitively on the set of nine 2-spaces in $V$ which do not contain $W$, so if $K$ is the stabilizer of such a 2-space then $[M: K \cap M]=9$. The stabilizers in $G$ of 1 -spaces from $V$ are conjugate in $\operatorname{Aut}(G)$ with the stabilizers of 2-spaces. We now see that if $X$ and $Y$ are distinct subspace stabilizers then $[X: X \cap Y] \in\{4,9,12\}$. In particular, $X \cap Y$ is not of prime index in $X$. Thus if $X_{0}<\ldots<X_{d-1}<X_{d}$ and $Y_{0}<\ldots<Y_{d-1}<Y_{d}$ are facets of $\Delta^{(d, d)}$ with $X_{d} \neq Y_{d}$, then $X_{d-1} \neq Y_{d-1}$. It now follows from Definition 1.1 that $\Delta^{(d, d)}$ is not shellable. Indeed, if $\sigma_{1}, \ldots, \sigma_{r}$ is a list of the facets, let $i$ be the smallest index such that the maximum element of $\sigma_{i}$ is not the maximum element of $\sigma_{1}$. Then $\left|\sigma_{i} \cap \sigma_{j}\right|<\left|\sigma_{i}\right|-1$ for all $j<i$, and this list is not a shelling.

The proof of Lemma 3.13 completes the proof of Theorem 3.2 Note that many of the results in this section can be obtained in the same manner as Lemma 3.13 that is, by examining intersections of maximal subgroups of a minimal simple group $G$ and showing that the complex $\Delta^{(d, d)}$ cannot be shellable. The proofs used here were chosen because they give some insight into the homotopy type and homology of $\Delta(\overline{\mathcal{L}(G)})$. We close this section by noting one further result.

Proposition 3.14. Let $G$ be a minimal simple group. Then $\tilde{H}_{1}(\Delta(\overline{\mathcal{L}(G)})) \neq 0$.

Proof. Given Lemmas 3.8 and 3.11, it is now sufficient to prove the claim when $G=S L_{3}(3)$. Using information given in [3], we see that $\Delta(\overline{\mathcal{L}(G)})$ contains facets of the form $X<Y$ with $X \cong Z_{3}$ and $Y \cong 13$.3. Let $\Delta_{0}$ be the subcomplex generated 
by these facets. Again using [3], we see that $\Delta_{0}$ is a graph with $\frac{|G|}{18}+\frac{|G|}{39}$ vertices and $\frac{|G|}{3}$ edges. Thus $\Delta_{0}$ is not a forest, so $\tilde{H}_{1}\left(\Delta_{0}\right) \neq 0$ and we are done.

\section{Solvable Groups ARe Shellable}

In this section, it will be shown that if $G$ is a solvable finite group then $\mathcal{L}(G)$ admits a recursive coatom ordering. It then follows from Theorem 2.14 that $\Delta(\overline{\mathcal{L}(G)})$ is shellable, and this completes the proof of Theorem 1.4. The reader who has read [18] should be aware that the proposed counterexample to the claim that all solvable finite groups are shellable given in that paper is incorrect, as a dihedral subgroup of order ten has been omitted from the subgroup lattice of the given group.

We will need some facts about the intersections of maximal subgroups of solvable groups. Recall that if $H$ is a subgroup of a finite group $G$ then $\operatorname{Core}_{G}(H)$ is the largest normal subgroup of $G$ contained in $H$.

Theorem 4.1 (Ore). Let $L$ and $M$ be maximal subgroups of a finite solvable group $G$. Then $L$ and $M$ are conjugate in $G$ if and only if $\operatorname{Core}_{G}(L)=\operatorname{Core}_{G}(M)$.

Proof. See Theorem (16.1) of section A of [5].

It follows immediately from the theorem above that the partial order described in the following definition is well-defined.

Definition 4.2. Let $G$ be a finite solvable group. For $H \leq G, H^{G}$ will denote the conjugacy class of $H$ in $G$. Define a partial order $\preceq$ on the conjugacy classes of maximal subgroups of $G$ by $L^{G} \preceq M^{G}$ if and only if $\operatorname{Core}_{G}(L) \leq \operatorname{Core}_{G}(M)$.

Theorem 4.3. Let $L$ and $M$ be maximal subgroups of a finite solvable group $G$ which are not conjugate in $G$. If $M^{G} \npreceq L^{G}$, then $L \cap M$ is a maximal subgroup of $L$.

Proof. This is Theorem (16.6) of Section A of [5].

Lemma 4.4. Let $M, M^{\prime}$ be conjugate maximal subgroups of a finite solvable group $G$, with $M \neq M^{\prime}$. Let $J$ be any proper subgroup of $M$ containing $M \cap M^{\prime}$. Then there exists some maximal subgroup $L<G$ such that $J \leq L$ and $M^{G} \prec L^{G}$.

Proof. Let $C=$ Core $_{G}(M)=$ Core $_{G}\left(M^{\prime}\right)$. There exists a unique subgroup $K \leq G$ such that $C<K \unlhd G, M K=M^{\prime} K=G$ and $M \cap K=M^{\prime} \cap K=C$ (see (15.2) of Section A of [5]). An order argument shows that $J K<G$. Let $L$ be any maximal subgroup of $G$ containing $J K$. Since $K \leq L$, we have $C<K \leq \operatorname{Core}_{G}(L)$, so $M^{G} \prec L^{G}$.

Definition 4.5. Let $G$ be a finite solvable group. An SC-list for $G$ is an ordering $\mathcal{M}$ of the maximal subgroups of $G$ with the following properties.

(1) If $L, M$ are maximal subgroups of $G$ which are not conjugate in $G$ and $L$ appears before $M$ in $\mathcal{M}$, then every element of $L^{G}$ appears before $M$ in $\mathcal{M}$.

(2) If $L, M$ are maximal subgroups of $G$ and $M^{G} \prec L^{G}$, then $L$ appears before $M$ in $\mathcal{M}$.

Note that condition (1) of Definition 4.5 requires that the conjugates of any maximal subgroup of $G$ form a segment in any SC-list for $G$. Also, it follows immediately from the fact that the partial order $\preceq$ has a linear extension that an SC-list for $G$ exists for any finite solvable group $G$. We can construct segments 
by listing the elements of each conjugacy class of maximal subgroups in arbitrary order and then get an SC-list by ordering the segments according to the reverse of some linear extension.

Lemma 4.6. Let $G$ be a finite solvable group and let $\mathcal{M}$ be an $S C$-list for $G$. Then $\mathcal{M}$ satisfies condition (T) of Proposition 2.13.

Proof. We must show that if $L$ appears before $M$ in $\mathcal{M}$ then there exists some $K$ appearing before $M$ in $\mathcal{M}$ such that $L \cap M \leq K \cap M$ and $K \cap M$ is maximal in $M$. First assume that $L$ and $M$ are not conjugate in $G$. Since $L$ appears before $M$ in $G, L^{G} \npreceq M^{G}$ and $L \cap M$ is maximal in $M$ by Theorem 4.3. Thus we can take $K=L$. Now assume $L$ and $M$ are conjugate in $G$. Let $J$ be any maximal subgroup of $M$ containing $L \cap M$. By Lemma 4.4 there exists some maximal $K<G$ such that $J \leq K$ and $M^{G} \prec K^{G}$. By condition (2) of Definition 4.5 $K$ appears before $M$ in $\mathcal{M}$. Since $J$ is maximal in $M, K \cap M=J$.

We will now see that we can choose SC-lists for all nontrivial subgroups of a finite group $G$ in a manner that satisfies condition $(\mathrm{R})$ of definition 2.12 .

Lemma 4.7. Let $G$ be a finite solvable group and let $\mathcal{M}=M_{1}, \ldots, M_{r}$ be an $S C$ list for $G$. Then for any $j \in[r]$, the set of maximal subgroups of $M_{j}$ of the form $M_{i} \cap M_{j}$ with $i<j$ is a union of $M_{j}$-conjugacy classes.

Proof. We must show that if $H=M_{i} \cap M_{j}$ is maximal in $M_{j}$ and $i<j$, then for every $x \in M_{j}$ there exists some $k<j$ such that $H^{x}=M_{k} \cap M_{j}$. If $M_{i}$ is conjugate to $M_{j}$ in $G$, then by Lemma 4.4 there exists some $M_{l}$ such that $H<M_{l}$ and $M_{j}^{G} \prec M_{l}^{G}$. Since $M_{l}$ is not conjugate to $M_{j}$ in $G$ and $\mathcal{M}$ is an SC-list, $l<j$. Since $H$ is maximal in $M_{j}, M_{l} \cap M_{j}=H$. Therefore, we may assume without loss of generality that $M_{i}$ and $M_{j}$ are not conjugate in $G$. Now for any $x \in M_{j}$ we have

$$
H^{x}=\left(M_{i} \cap M_{j}\right)^{x}=M_{i}^{x} \cap M_{j} .
$$

Since $\mathcal{M}$ is an SC-list and $M_{i}$ appears before $M_{j}$ in $\mathcal{M}$ with $M_{i}$ and $M_{j}$ not conjugate in $G, M_{i}^{x}$ appears before $M_{j}$ in $\mathcal{M}$.

Lemma 4.8. Let $G$ be a finite solvable group and let $\mathcal{M}=M_{1}, \ldots, M_{r}$ be an $S C$ list for $G$. Fix $j \in[r]$. Let $H=M_{i} \cap M_{j}$ be a maximal subgroup of $M_{j}$ with $i<j$. Let $X$ be a maximal subgroup of $M_{j}$ with $\operatorname{Core}_{M_{j}}(H) \leq$ Core $_{M_{j}}(X)$. Then there is some $l<j$ such that $X=M_{l} \cap M_{j}$.

Proof. As in the proof of Lemma 4.7, we may assume that $M_{i}$ and $M_{j}$ are not conjugate in $G$. Now

$$
\text { Core }_{G}\left(M_{i}\right) \cap M_{j} \leq \text { Core }_{M_{j}}(H) \leq \text { Core }_{M_{j}}(X) \leq X,
$$

so $\operatorname{Core}_{G}\left(M_{i}\right) \cap M_{j}=\operatorname{Core}_{G}\left(M_{i}\right) \cap X$. An order argument gives $X \operatorname{Core}_{G}\left(M_{i}\right)<$ $G$. Let $M_{l}$ be any maximal subgroup of $G$ containing $X \operatorname{Core}_{G}\left(M_{i}\right)$. Since $X$ is maximal in $M_{j}, M_{l} \cap M_{j}=X$. We now have

- $i<j$,

- $\operatorname{Core}_{G}\left(M_{i}\right) \leq \operatorname{Core}_{G}\left(M_{l}\right)$,

- $M_{i}$ and $M_{j}$ are not conjugate in $G$, and

- $\mathcal{M}$ is an SC-list for $G$.

It follows that $l<j$. 
Corollary 4.9. Let $G$ be a finite solvable group and let $\mathcal{M}=M_{1}, \ldots, M_{r}$ be an $S C$-list for $G$. Then for each $j \in[r]$ there exists an $S C$-list for $M_{j}$ in which all maximal subgroups of $M_{j}$ of the form $M_{i} \cap M_{j}$ with $i<j$ appear before all other maximal subgroups of $M_{j}$.

Proof. Fix $j$ and let $\mathcal{J}_{1}$ be the set of all maximal subgroups of $M_{j}$ of the form $M_{i} \cap M_{j}$ with $i<j$, and let $\mathcal{J}_{2}$ be the set of all the other maximal subgroups of $M_{j}$. By Lemma 4.7 each $\mathcal{J}_{i}$ is a union of $M_{j}$-conjugacy classes. Let $\preceq_{j}$ be the partial order on conjugacy classes of maximal subgroups of $M_{j}$ as given in Definition 4.2. Using the restriction of $\preceq_{j}$ to the $M_{j}$-conjugacy classes contained in $\mathcal{J}_{i}$, we see that for each $i \in\{1,2\}$ there exists a list $\mathcal{R}_{i}$ of the elements of $\mathcal{J}_{i}$ satisfying the following conditions.

(a) If $X, Y \in \mathcal{J}_{i}$ with $X$ and $Y$ not conjugate in $M_{j}$ and $X$ appears before $Y$ in $\mathcal{R}_{i}$, then every $M_{j}$-conjugate of $X$ appears before $Y$ in $\mathcal{R}_{i}$.

(b) If $X, Y \in \mathcal{J}_{i}$ and $X^{M_{j}} \preceq_{j} Y^{M_{j}}$, then $Y$ appears before $X$ in $\mathcal{R}_{i}$.

Let $\mathcal{R}$ be the list of maximal subgroups of $M_{j}$ obtained by placing $\mathcal{R}_{2}$ after $\mathcal{R}_{1}$. Given the fact that each $\mathcal{R}_{i}$ satisfies conditions (a) and (b) above, it is sufficient to show that if $Y \in \mathcal{J}_{2}$ and $X \in \mathcal{J}_{1}$ then $X \swarrow_{j} Y$. This follows immediately from Lemma 4.8 .

Lemma 4.6 and Corollary 4.9 give the following result.

Corollary 4.10. If $G$ is a finite solvable group, then $\mathcal{L}(G)$ admits a recursive coatom ordering.

By Theorem 2.14, $\mathcal{L}(G)$ is shellable, and the proof of Theorem 1.4 is complete.

\section{REFERENCES}

1. A. Björner, Shellable and Cohen-Macaulay partially ordered sets, Trans. Amer. Math. Soc. 260 (1980), 159-183. MR 81:06001

2. A. Björner and M. Wachs, Shellable nonpure complexes and posets, I, Trans. Amer. Math. Soc. 348 (1996), 1299-1327. MR 96i:06008

3. J. H. Conway, R. T. Curtis, S. P. Norton, R. A. Parker and R. A. Wilson, "Atlas of finite groups. Maximal subgroups and ordinary characters for simple groups. With computational assistance from J. G. Thackray", Oxford University Press, Oxford, 1985. MR 88g:20025

4. L. E. Dickson, "Linear Groups with an Exposition of the Galois Theory", Dover, New York, 1984. MR 21:3488 (earlier ed.)

5. K. Doerk and T. Hawkes, "Finite Soluble Groups", de Gruyter Expositions in Mathematics 4, Walter de Gruyter, Berlin, 1992. MR 93k:20033

6. M. Downs, "Möbius inversion of some classical groups and their application to the enumeration of regular maps", Ph.D. Thesis, University of Southampton, 1988.

7. M. Downs, The Möbius function of $P S L_{2}(q)$, with an application to the maximal normal subgroups of the modular group, J. London Math. Soc. (2) 43 (1991), 61-75. MR 92d:20071

8. L. C. Glaser, "Geometrical Combinatorial Topology", vol. 1, Van Nostrand, New York, 1970.

9. P. Hall, The Eulerian functions of a group, Quart. J. Math. 7 (1936), 134-151.

10. K. Iwasawa, Über die endlichen Gruppen und die Verbände ihrer Untergruppen, J. Fac. Sci. Imp. Univ. Tokyo 4 (1941), 171-199. MR 3:193h

11. C. Kratzer and J. Thévenaz, Type d'homotopie des treillis et treillis des sous-groupes d'un groupe fini, Comment. Math. Helv. 60 (1985), 85-106. MR 87b:06017

12. D. Quillen, Homotopy Properties of the Poset of Nontrivial $p$-Subgroups of a Group, Advances in Math. 28 (1978), 101-128. MR 80k:20049

13. J. Shareshian, "Combinatorial properties of subgroup lattices of finite groups", Ph.D. Thesis, Rutgers University, 1996.

14. R. Stanley, Supersolvable lattices, Algebra Universalis 2 (1972), 197-217. MR 46:8920 
15. M. Suzuki, On a class of doubly transitive groups, Annals of Math. 75 (1962), 105-145. MR 25:112

16. J. Thévenaz, The top homology of the lattice of subgroups of a solvable group, Discrete Math. 55 (1985), 291-303. MR 86j:20045

17. J. G. Thompson, Nonsolvable finite groups all of whose local subgroups are solvable, Bull. Amer. Math. Soc. 74 (1968), 383-437. MR 37:6367

18. V. Welker, Shellability in the lattice of subgroups of a finite group, in "Jerusalem Combinatorics '93", Contemp. Math. 178, Amer. Math. Soc., Providence, RI, 1994, pp. 335-360. MR 95k:06014

California Institute of Technology, Pasadena, California 91125

Current address: Department of Mathematics, University of Miami, Coral Gables, Florida 33124

E-mail address: shareshi@math.miami.edu 